\title{
FINDING THE EXACT SOLITON SOLUTIONS OF NONLINEAR FOURTH-ORDER EQUATION OF THE GENERALIZED HIROTA-SATSUMA COUPLED WITH KdV
}

\author{
GH. FOROZANI and B. SOHRABI \\ Department of Physics \\ Payame Noor University \\ P. O. Box 19395-3697 \\ Tehran \\ Iran \\ e-mail: g.forozani@gmail.com \\ Department of Physics \\ Faculty of Science \\ Bu-Ali Sina University \\ 6517838695, Hamedan \\ Iran
}

\begin{abstract}
In this article, the generalized fourth-order Hirota-Satsuma partial differential equation coupled with KdV system is investigated by using the tanh method. In this method, by choosing an independent variable, the Hirota Satsuma partial differential equations (PDEs) change into ordinary differential equations (ODEs). These equations have been solved and the soliton solutions are obtained.
\end{abstract}

2010 Mathematics Subject Classification: 35Q51, 35G20.

Keywords and phrases: fourth-order generalized Hirota-Satsuma, KdV equation, tanh method, soliton wave.

Received July 11, 2014

(ㄷ) 2015 Scientific Advances Publishers 


\section{Introduction}

Research on solitary waves and soliton solutions, was first conducted by Russell John Scott (1808-1882) when he followed the path of a solitary wave at a canal [1]. To define the soliton, it can not be considered as a singular meaning. Solutions of nonlinear wave equations which have the following three properties are called soliton:

(1) Their shape and velocity are not changed.

(2) Is confined in a region of space.

(3) After the collision with other solitons, its shape is preserved.

Answers that include the first property, have been called solitary waves. Inherent stability of solitons, enable them to be sent over long distances without the use of repeaters and could potentially double transmission capacity. Soliton waves are quite stable, and in case of disturbance continue to move to its initial state.

After Scott, more than a century, the solitons were not heeded. Then in 1965, Norman Zabvsky, from Bell Lab and Martin Kruskal of Princeton University, who described the behaviour of solitons in the form of mathematics. Since then, gradually, solitons were used not only to describe water waves, but also in other fields of physics that deal with the wave and showed excellent performance [2].

Nonlinear partial differential equations (PDEs) in different scientific fields such as fluid mechanics, solid state physics, plasma physics, chemical physics [3], and so on are of high importance. Completely integrable nonlinear partial differential equations (PDEs) model physically interesting wave phenomena in reaction-diffusion systems, population and molecular dynamics, nonlinear networks, chemical reactions, and waves in material science [4]. Finding exact responses to these equations will help us to better understanding of our environmental nonlinear physical phenomena. For most nonlinear partial 
differential equations, soliton solutions can be defined. One of these equations is nonlinear equation of generalized Hirota-Satsuma coupled with a KdV system, which will be shown below:

$$
\begin{aligned}
u_{t} & =\frac{1}{4} u_{x x x}+3 u u_{x}+3\left(-v^{2}+w\right)_{x} \\
v_{t} & =-\frac{1}{2} v_{x x x}-3 u v_{x} \\
w_{t} & =-\frac{1}{2} w_{x x x}-3 u w_{x}
\end{aligned}
$$

The discussed generalized Hirota-Satsuma coupled KdV equations have been studied by many authors via different approaches. Recently, Fan [5] has provided a suggestion to construct soliton solutions for Equations (1), (2), and (3) by using an extended tanh-function method and symbolic computation. The main idea of this method is to take full advantage of a Riccati equation involving a parameter and to use its solutions to replace the tanh-function method. Following him, Jacobi elliptic function method by Yu et al. [6], the projective Riccati equations method by Yong and Zhang [7], the algebraic method by Zayed et al. [8], variational iteration method by $\mathrm{He}$ and $\mathrm{Wu}$ [9] and Assas [10], Adomians decomposition method by Kaya [11] and Raslan [12], homotopy perturbation method by Ganji and Rafei [13], homotopy analysis method by Abbasbandy [14], homogeneous balance method by Yong et al. [15], Jacobian and rational methods by Zayed et al. [16], and the trigonometric function transform method by Cao et al. [17].

If in Equation (1), $w$ does not depend on $x$ and $t$, then $w_{x}=0$, $w_{t}=0$. In fact, the above mentioned equations are transformed into the following equations:

$$
\begin{aligned}
& u_{t}=\frac{1}{4} u_{x x x}+3 u u_{x}-6 v v_{x} \\
& v_{t}=-\frac{1}{2} v_{x x x}-3 u v_{x}
\end{aligned}
$$


Equations (4) and (5) describe an interaction of two long waves with different dispersion relations [18]. For such a special behaviour, what we mention here is that some coupled nonlinear systems usually exhibit special interesting dynamics. For example, the Hirota-Satsuma equation, which has two potential functions, $u$ and $v$. The main potential $u$ admits a sudden shift in scattering, and this invisible interaction is considered to be caused by another potential $v[19]$.

In this article, we solve the fourth-order of this equation, which is defined as follows:

$$
\begin{aligned}
& u_{t}=u_{x x x x}+\frac{1}{4} u_{x x x}+3 u u_{x}-6 v v_{x} \\
& v_{t}=v_{x x x x}+\frac{1}{2} v_{x x x}+3 u v_{x} .
\end{aligned}
$$

That $u(x, t)$ and $v(x, t)$ are solutions of the above equations in one space and time dimension, namely, $(1+1)$ dimensions and; $u_{t}(x, t)=$

$$
\begin{aligned}
& \frac{\partial u(x, t)}{\partial t} ; u_{x}(x, t)=\frac{\partial u(x, t)}{\partial x} ; u_{x x x}(x, t)=\frac{\partial^{3} u(x, t)}{\partial x^{3}} ; u_{x x x x}(x, t)=\frac{\partial^{4} u(x, t)}{\partial x^{4}} ; \\
& v_{t}(x, t)=\frac{\partial v(x, t)}{\partial t} ; v_{x}(x, t)=\frac{\partial v(x, t)}{\partial x} ; v_{x x x}(x, t)=\frac{\partial^{3} v(x, t)}{\partial x^{3}} ; v_{x x x x}(x, t)=\frac{\partial^{4} v(x, t)}{\partial x^{4}} .
\end{aligned}
$$

Of course, there are different methods for finding soliton solutions of Equations (6) and (7). Some of these methods include:

(1) Backlund transformation [20].

(2) Darboux transformation [21].

(3) Tanh-function [22, 23].

(4) Extended tanh-function [24].

(5) Sine-cosine [25].

(6) Lie group analysis [26] and so on. In this article, the tanh-function method is used. 


\section{Tanh Method}

In this section, we find analytical solutions to Equations (6) and (7) by using the hyperbolic tangent method. This method is proven very well at finding soliton solutions because many soliton solutions can be written as hyperbolic tangent functions. It is necessary to remember that since many nonlinear equations using hyperbolic tangent method has been solved and studied by Wazwaz, this method is called Wazwaz method.

The partial differential equation (6) and (7) can be converted to an ordinary differential equation upon using a wave variable $z=x-c t$ :

$$
\begin{aligned}
& \frac{d^{4} U(z)}{d z^{4}}+\frac{1}{4} \frac{d^{3} U(z)}{d z^{3}}+3 U(z) \frac{d U(z)}{d z}+c \frac{d U(z)}{d z}-6 V(z) \frac{d V(z)}{d z}=0 \\
& \frac{d^{4} V(z)}{d z^{4}}-\frac{1}{2} \frac{d^{3} V(z)}{d z^{3}}-3 U(z) \frac{d V(z)}{d z}+c \frac{d V(z)}{d z}=0
\end{aligned}
$$

That $u(x, t)=U(z), v(x, t)=V(z)[27]$.

Again, we introduce another independent variable in the form $y=\operatorname{Tanh} z$ which will lead to derivative changes as follows [28]:

$$
\begin{gathered}
\frac{d}{d z}=\left(1-y^{2}\right) \frac{d}{d y}, \\
\frac{d^{2}}{d z^{2}}=\left(1-y^{2}\right)\left[-2 y \frac{d}{d y}+\left(1-y^{2}\right) \frac{d^{2}}{d z^{2}}\right] \\
\frac{d^{3}}{d z^{3}}=\left(1-y^{2}\right)\left[\left(6 y^{2}-2\right) \frac{d}{d y}-6 y\left(1-y^{2}\right) \frac{d^{2}}{d y^{2}}+\left(1-y^{2}\right)^{2} \frac{d^{3}}{d y^{3}}\right], \\
\frac{d^{4}}{d z^{4}}=\left(1-y^{2}\right)\left[8 y\left(3 y^{2}-2\right) \frac{d}{d y}+4\left(1-y^{2}\right)\left(9 y^{2}-2\right) \frac{d^{2}}{d y^{2}}\right. \\
\left.-12 y\left(1-y^{2}\right)^{2} \frac{d^{3}}{d y^{3}}+\left(1-y^{2}\right)^{3} \frac{d^{4}}{d y^{4}}\right] .
\end{gathered}
$$


With substituting (10) to (13), in Equations (8) and (9), the result is

$$
\begin{gathered}
\left(1-y^{2}\right)^{3} \frac{d^{4} U(y)}{d y^{4}}+\left(1-y^{2}\right)^{2}\left(-12 y+\frac{1}{4}\right) \frac{d^{3} U(y)}{d y^{3}}+\left(1-y^{2}\right)\left(36 y^{2}-6 y-8\right) \frac{d^{2} U(y)}{d y^{2}} \\
+\left(-24 y^{3}+6 y^{2}+16 y-2+3 U(y)+c\right) \frac{d U(y)}{d y}-6 V(y) \frac{d V(y)}{d y}=0, \\
\left(1-y^{2}\right)^{3} \frac{d^{4} V(y)}{d y^{4}}+\left(1-y^{2}\right)^{2}\left(-12 y+\frac{1}{2}\right) \frac{d^{3} V(y)}{d y^{3}}+\left(1-y^{2}\right)\left(36 y^{2}-3 y-8\right) \frac{d^{2} V(y)}{d y^{2}} \\
+\left(-24 y^{3}-3 y^{2}+16 y+1-3 U(y)+c\right) \frac{d V(y)}{d y}=0
\end{gathered}
$$

For a detailed response, limited extension of $y$ is considered as following:

$$
U(y)=\sum_{m=0}^{M} a_{m} y^{m}, \quad V(y)=\sum_{n=0}^{N} b_{n} y^{n} .
$$

Placement of (16) and (10) to (13) in Equations (14) and (15) and embed the highest level of linear order with the highest level of nonlinear order, is determined that

$$
M=N=3 .
$$

With placement of these numbers in the series of Equation (16) the following equations are obtained:

$$
\begin{aligned}
& U(y)=a_{0}+a_{1} y+a_{2} y^{2}+a_{3} y^{3}, \\
& V(y)=b_{0}+b_{1} y+b_{2} y^{2}+b_{3} y^{3} .
\end{aligned}
$$

Then, Equations (18) and (19) and their derivatives in Equations (14) and (15) are inserted. If the coefficients of each power of $y$ are added and equalled to zero, the following equations will be obtained:

$$
\begin{gathered}
a_{3}^{2}-40 a_{3}-2 b_{3}^{2}=0, \\
3.7 a_{3}-8 a_{2}+a_{2} a_{3}-2 b_{2} b_{3}=0 \\
76 a_{3}+4 a_{2}-4 a_{1}+a_{2}^{2}+2 a_{1} a_{3}-4 b_{1} b_{3}-2 b_{2}^{2}=0,
\end{gathered}
$$




$$
\begin{gathered}
-15 a_{3}+40 a_{2}+2 a_{1}+3 a_{1} a_{2}+3 a_{0} a_{3}-6 b_{1} b_{2}-6 b_{0} b_{3}+a_{3} c=0 \\
-120 a_{3}-16 a_{2}+16 a_{1}+3 a_{1}^{2}+6 a_{0} a_{2}-6 b_{1}^{2}-12 b_{0} b_{2}+2 a_{2} c=0 \\
3 a_{3}-32 a_{2}-4 a_{1}+6 a_{0} a_{1}-12_{0} b_{1}+2 a_{1} c=0 \\
40 b_{3}+a_{3} b_{3}=0 \\
-20 b_{3}-120_{2}-6 a_{3} b_{2}-9 a_{2} b_{3}=0 \\
120 b_{3}-4 b_{2}-8 b_{1}-a_{3} b_{1}-2 a_{2} b_{2}-3 a_{1} b_{3}=0 \\
27 b_{3}+88 b_{2}-3 b_{1}-3 a_{2} b_{1}-6 a_{1} b_{2}-9 a_{0} b_{3}-3 b_{3} c=0 \\
-120 b_{3}+8 b_{2}+16 b_{1}-3 a_{1} b_{1}-6 a_{0} b_{2}+2 b_{2} c=0 \\
-3 b_{3}-16 b_{2}+b_{1}-3 a_{0} b_{1}+b_{1} c=0 .
\end{gathered}
$$

Using Mathematica software, we can solve Equations (20) to (31), so the coefficients can be found as follows:

\section{Case 1.}

$$
\begin{gathered}
a_{0}=415.37, \quad a_{1}=30.63, \quad a_{2}=-2.365, \quad a_{3}=-40, \quad b_{0}=-414.21, \\
b_{1}=-35.1, \quad b_{2}=-0.43, \quad b_{3}=40, \quad c=1214.9 . \quad(32)
\end{gathered}
$$

By placing these coefficients in Equations (18) and (19) soliton solutions can be obtained as follows, of course $y=\operatorname{Tanh} z$ and $z=x-c t$ :

$$
\begin{aligned}
& u_{1}(x, t)=415.37+30.63 \tanh (x-1241.9 t)-2.365 \tanh ^{2}(x-1241.9 t) \\
& -40 \tanh ^{3}(x-1241.9 t), \\
& v_{1}(x, t)=-414.21-35.1 \tanh (x-1241.9 t)-0.43 \tanh ^{2}(x-1241.9 t) \\
& +40 \tanh ^{3}(x-1241.9 t) \text {. }
\end{aligned}
$$

The numerical simulation of two solitons $u_{1}$ and $v_{1}$ are presented in the following: 


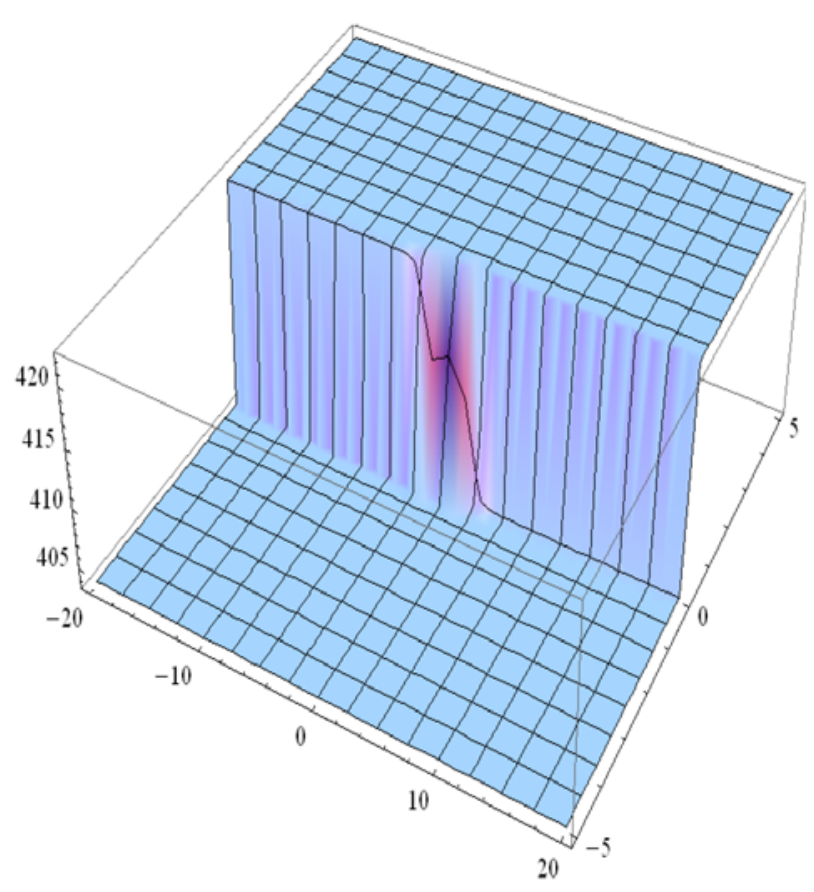

Figure 1. $u_{1}(x, t)$.

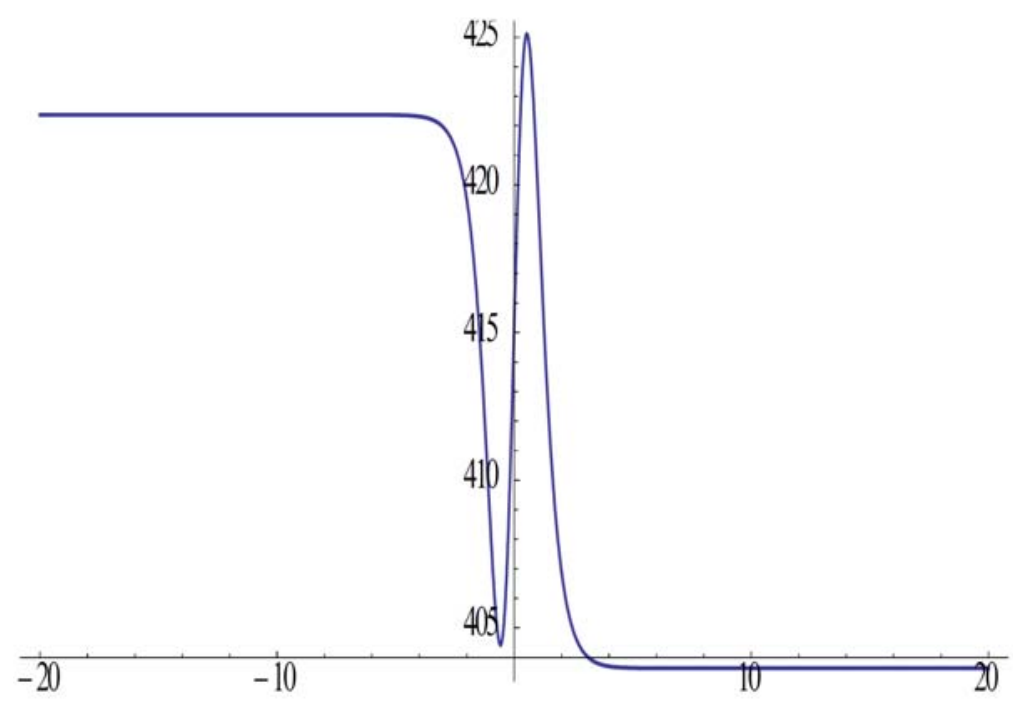

Figure 2. $u_{1}(x, t)$ when $t=0$. 


\section{Case 2.}

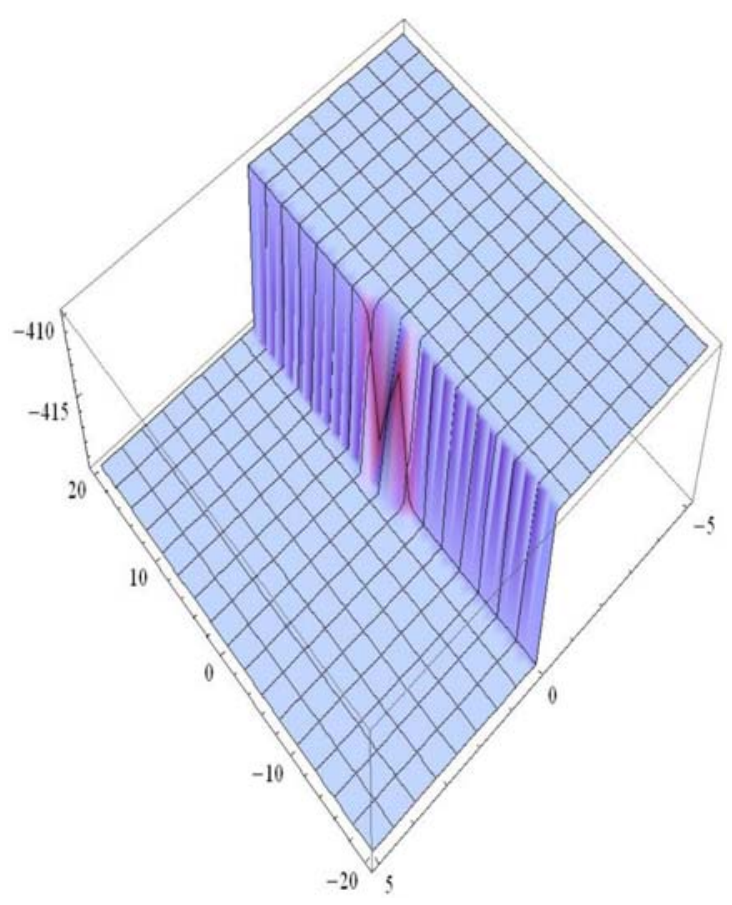

Figure 3. $v_{1}(x, t)$.

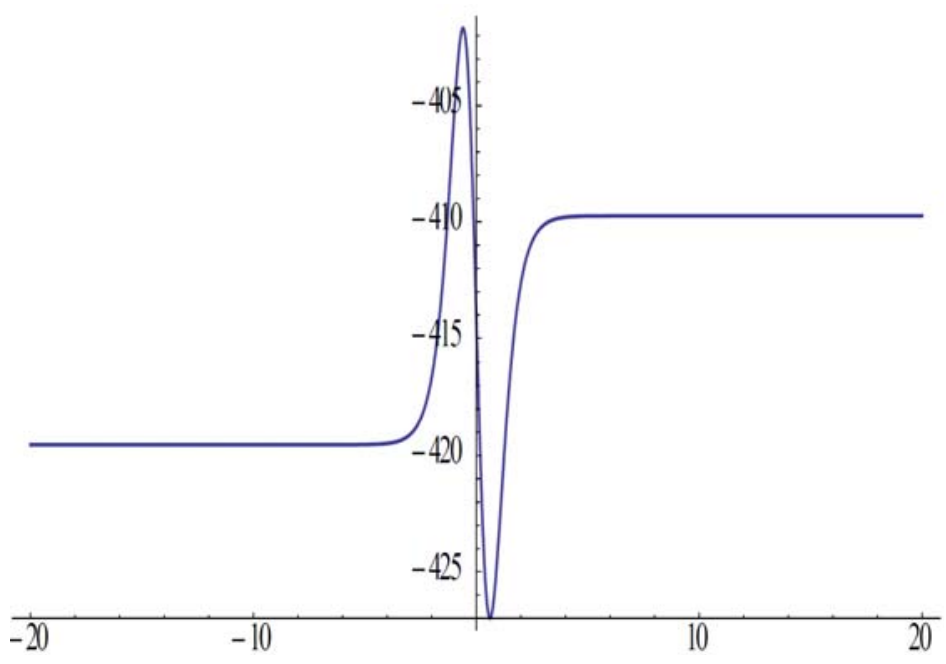

Figure 4. $v_{1}(x, t)$ when $t=0$. 
If it is assumed that $a_{1}=b_{3}=0$ :

$$
\begin{aligned}
a_{0}=-16.785, \quad a_{2}=-4.625, & a_{3}=40, \\
b_{0}=5.23, \quad b_{1}=4.265, \quad b_{2}=39, & c=94.93 .
\end{aligned}
$$

By placing these coefficients in Equations (18) and (19) soliton solutions can be obtained as follows, of course $y=\operatorname{Tanh} z$ and $z=x-c t$ :

$$
\begin{gathered}
u_{2}(x, t)=-16.785-4.625 \tanh ^{2}(x-94.93 t)+40 \tanh ^{3}(x-9493 t) \\
v_{2}(x, t)=5.23+4.265 \tanh (x-94.93 t)+39 \tanh ^{2}(x-94.93 t)
\end{gathered}
$$

The numerical simulation of two solitons $u_{2}$ and $v_{2}$, are presented in the following figures:

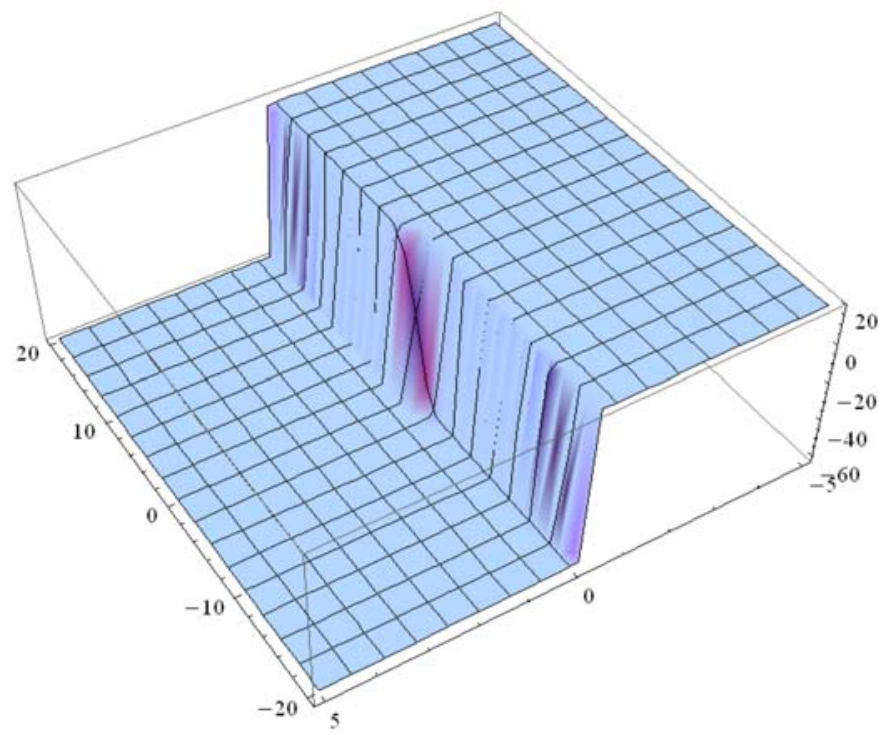

Figure 5. $u_{2}(x, t)$. 
FINDING THE EXACT SOLITON SOLUTIONS ...

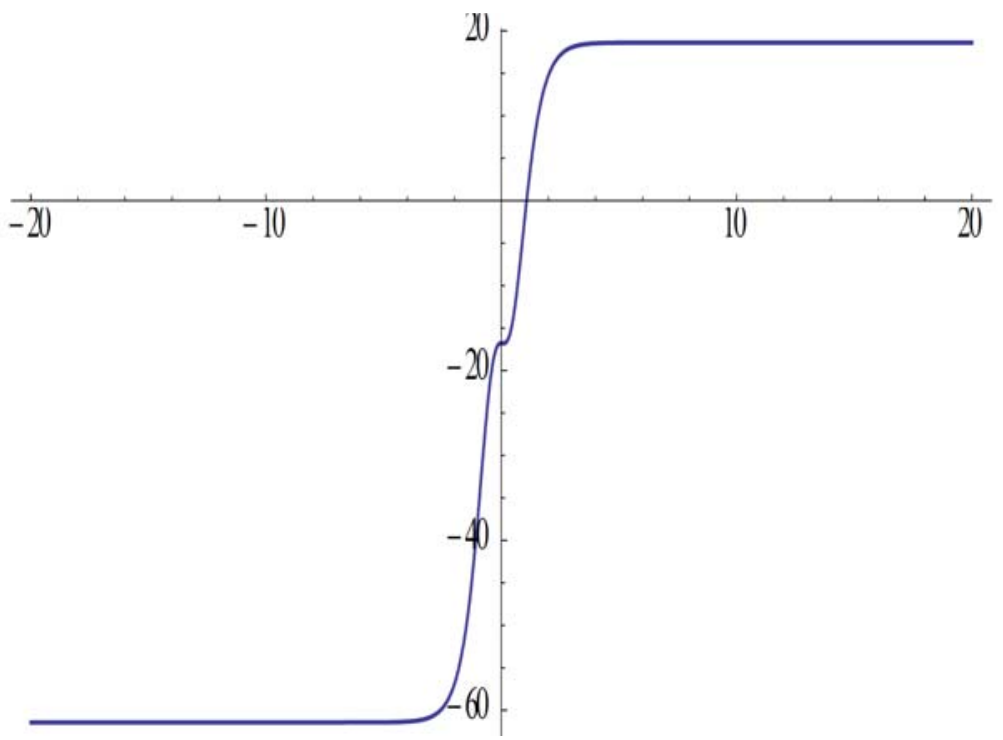

Figure 6. $u_{2}(x, t)$ when $t=0$.

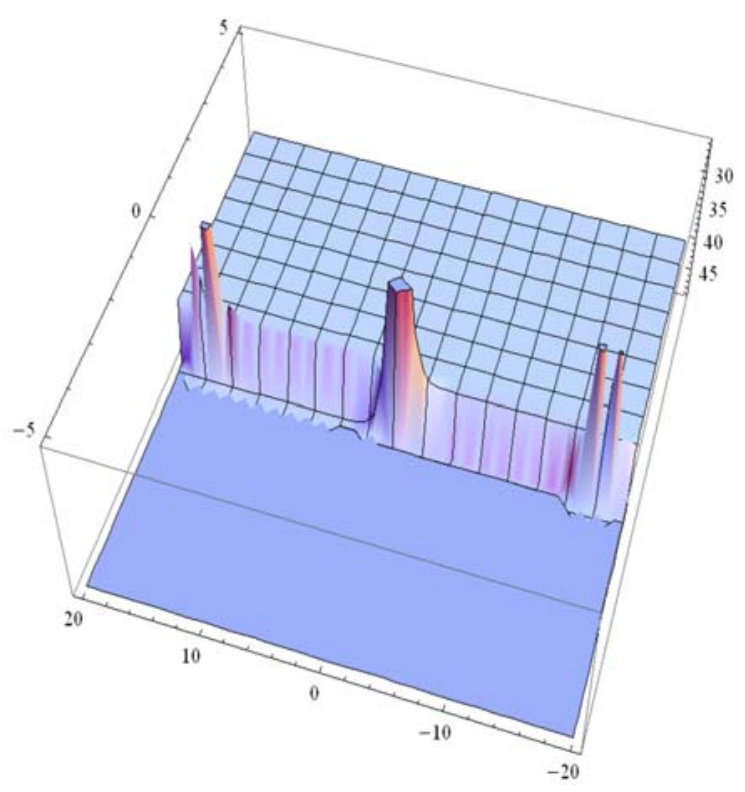

Figure 7. $v_{2}(x, t)$. 


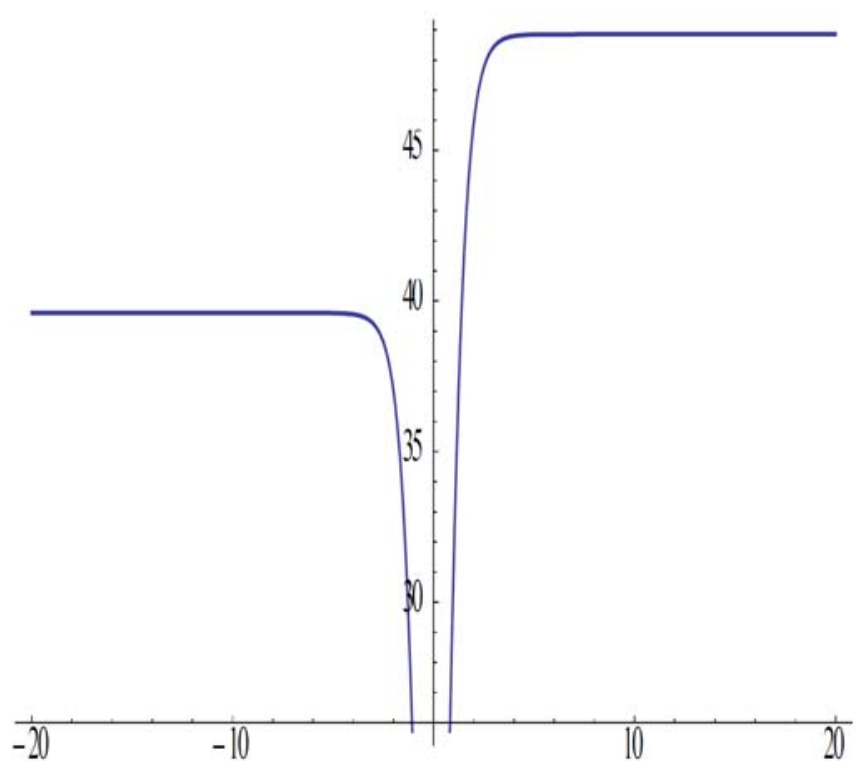

Figure 8. $v_{2}(x, t)$ when $t=0$.

\section{Conclusion}

The main goal in this article was, to find the soliton solutions of nonlinear fourth-order equation of the generalized Hirota-Satsuma coupled with the KdV system. $(1+1)$ dimensional and hyperbolic tangent method that were used, since most forms of soliton equations are hyperbolic tangent functions, and the Mathematica software version 9 was used for drawing figures.

\section{Acknowledgement}

Author was supported by Department of Physics, Faculty of Science, Bu-Ali Sina University, Hamedan, Iran. 


\section{References}

[1] R. J. Scott, Report on Waves, Report of the fourteenth meeting of the British Association for the Advancement of Science, York, September, 1844.

[2] H. D. Wahlquist and F. B. Estabrook, Prolongation structures of nonlinear evolution equations II, J. Math. Phys. 17 (1976), 1293-1297.

[3] M. E. Khalifa and M. Elgamal, Appl. Math. Comput. 160 (2005), 451.

[4] D. Baldwin and W. Hereman, Symbolic software for the Painleve test of nonlinear ordinary and partial differential equations, Journal of Nonlinear Mathematical Physics 13 (2006), 90-110.

[5] E. G. Fan, Soliton solutions for a generalized Hirota-Satsuma coupled KdV equation and a coupled mKdV equation, Phys. Lett. A 282 (2001), 18-22.

[6] Y. Yu, Q. Wang and H. Zhang, The extended Jacobi elliptic function method to solve a generalized Hirota-Satsuma coupled KdV equations, Chaos, Solitons and Fractals 26 (2005), 1415-1421.

[7] X. L. Yong and H. Q. Zhang, New exact solutions to the generalized coupled HirotaSatsuma KdV system, Chaos, Solitons and Fractals 26 (2005), 1105-1110.

[8] E. M. E. Zayed, H. Zedan and K. A. Gepreel, On the solitary wave solutions for nonlinear Hirota-Satsuma coupled KdV of equations, Chaos, Solitons and Fractals 22 (2004), 285-303.

[9] J. H. He and X. H. Wu, Construction of solitary solution and compaction-like solution by variational iteration method, Chaos, Solitons and Fractals 29 (2006), 108-113.

[10] L. M. B. Assas, Variational iteration method for solving coupled-KdV equations, Chaos, Solitons and Fractals 38 (2008), 1225-1228.

[11] D. Kaya, Solitary wave solutions for a generalized Hirota-Satsuma coupled KdV equation, Appl. Math. Comput. 147 (2004), 69-78.

[12] K. R. Raslan, The decomposition method for a Hirota-Satsuma coupled KdV equation and a coupled mKdV equation, Int. J. Comput. Math. 81 (2004), 1497-1505.

[13] D. D. Ganji and M. Rafei, Solitary wave solutions for a generalized Hirota-Satsuma coupled KdV equation by homotopy perturbation method, Phys. Lett. A 356 (2006), 131-137.

[14] S. Abbassandy, The application of homotopy analysis method to solve a generalized Hirota-Satsuma coupled KdV equation, Phys. Lett. A 361 (2007), 478-483.

[15] C. Yong, Y. Zhen-Ya, L. Biao and Z. Hong-Qing, New explicit exact solutions for a generalized Hirota-Satsuma coupled KdV system and a coupled mKdV equation, Chinese Phys. 12 (2003), 1-10.

[16] E. M. E. Zayed, A. M. Abourabia, K. A. Gepreel and M. M. El Horbaty, Travelling solitary wave solutions for the nonlinear coupled Korteweg-de Vries system, Chaos, Solitons and Fractals 34 (2007), 292-306. 
[17] D. B. Cao, J. R. Yang and Y. Zhang, Exact solutions for a new coupled mKdV equations and a coupled KdV equations, Phys. Lett. A 297 (2002), 68-74.

[18] R. Hirota and J. Satsuma, Soliton solution of a coupled KdV equations, Phys. Lett. A 85 (1981), 407- 409.

[19] D. J. Zhanga, J. Ji and S. l. Zhao, Soliton scattering with amplitude changes of a negative order AKNS equation, Physica D 238 (2009), 2361-2367.

[20] C. Rogers and W. R. Shadwick, Backlund Transformations and their Applications, Academic, New York, 1982.

[21] V. B. Matveev and M. A. Salle, Darboux Transformations and Solitons, Springer, Berlin, 1991.

[22] T. C. Xia, B. Li and H. Q. Zhang, New explicit and exact solutions for the NizhnikNovikov-Vesselov equation, Appl. Math. E-Notes 1 (2001), 139-142.

[23] Gh. Forozani and M. Ghorveei Nosrat, Solitary solution of modified bad and good Bouessinesq equation by using of tanh and extended tanh methods, Indian J. Pure Appl. Math. 44 (2013), 497-510.

[24] E. G. Fan, Extended tanh-function method and its applications to nonlinear equations, Phys. Lett. A 277 (2000), 212-218.

[25] X. D. Zheng, T. C. Xia and H. Q. Zhang, New exact traveling wave solutions for compound KdV-Burgers equations in mathematical physics, Appl. Math. E-Notes 2 (2002), 45-50.

[26] D. Lu, B. Hong and L. Tian, New explicit exact solutions for the generalized coupled Hirota-Satsuma KdV system, Comput. Math. 53 (2007), 1181-1190.

[27] H. A. Zedan, New approach for tanh and extended-tanh methods with applications on Hirota-Satuma equations, Comput. Math. 28 (2009), 1-14.

[28] M. Alquran and R. Al-omary, Soliton and periodic solutions to the generalized Hirota-Satsuma coupled system using trigonometric and hyperbolic function methods, Academic Journal 14 (2012), 497-505. 